\title{
SU(2) Yang-Mills thermodynamics: a priori estimate and radiative corrections
}

\author{
Ralf Hofmann ${ }^{1, a}$ \\ ${ }^{1}$ Institut für Theoretische Physik, Universität Heidelberg, Philosophenweg 16, 69120 Heidelberg
}

\begin{abstract}
We review and explain essential characteristics of the a priori estimate of the thermal ground state and its excitations in the deconfining phase of SU(2) Quantum Yang-Mills thermodynamics. This includes the spatially central and peripheral structure of Harrington-Shepard (anti)calorons, a sketch on how a spatial coarse-graining over (anti)caloron centers yields an inert scalar field, which is responsible for an adjoint Higgs mechanism, the identification of (anti)caloron action with $\hbar$, a discussion of how, owing to (anti)caloron structure, the thermal ground state can be excited (wave-like and particlelike massless modes, massive thermal quasiparticle fluctuations), the principle role of and accounting for radiative corrections, the exclusion of energy-sign combinations due to constraints on momenta transfers in four-vertices in a completely fixed, physical gauge, dihedral diagrams and their resummation up to infinite loop order in the massive sector, and the resummation of the one-loop polarisation tensor of the massless modes. We also outline applications of deconfining SU(2) Yang-Mills thermodynamics to the Cosmic Microwave Background (CMB) which affect the cosmological model at high redshifts, the redshift for re-ionization of the Universe, the $\mathrm{CMB}$ angular power spectra at low $l$, and the late-time emergence of intergalactic magnetic fields.
\end{abstract}

\section{Introduction}

Non-Abelian gauge theory in four spacetime dimensions exhibits beautiful, rich, and deep structures with clear and convincing links to experimentally accessible, fundamental phenomena such as asymptotic freedom [1-5], chiral symmetry breaking [6, 7], confinement [8,9], the axial anomaly [10-12], and fundamental, theoretically prescribed [13-16] as well as adjoint, theoretically emergent [17] gauge-symmetry breaking.

Yang-Mills thermodynamics, a field theory formulated solely in terms of gauge potentials which live on the Euclidean cylinder $S_{1} \times \mathbf{R}^{3}$ of temporal extent $0 \leq x_{4} \leq \beta=\frac{1}{T}$, even when subjected to the simplest non-Abelian group SU(2) comprises complex ground states in each of its three phases deconfining, preconfining, and confining [18]. In particular, the thermal ground state of the deconfining phase is represented by (anti)selfdual gauge-field configurations of topological charge modulus unity and trivial holonomy - Harrington-Shepard (anti)calorons [19] - whose spatially densely packed centers and overlapping peripheries determine the nature of this ground state's quantum and classical excitations, respectively $[17,20]$. While (anti)caloron peripheries provide (anti)selfdual dipole

ae-mail: r.hofmann@thphys.uni-heidelberg.de 
fields, effectively creating electric and magnetic dipole densities whose undulating re-polarisations associate with the classical propagation of low-frequency electromagnetic waves [20], (anti)caloron centers associate with thermal quasiparticle fluctuations which are short-lived and local quantum, that is, indeterministic excitations whose energy and momentum are governed by the (anti)caloron action (Planck's quantum of action $\hbar[17,21]$ ). Depending on whether quantum excitations occur within or off the Cartan subalgebra, defined by the direction of the effective, adjoint, inert, and spatially homogeneous scalar field $\phi$ in the SU(2) algebra su(2), they are massless or exhibit a substantial mass gap (adjoint Higgs mechanism), respectively [17]. Notice that the field $\phi$ represents (anti)caloron centers (without packing voids) in a point-like way at a spatial resolution set by its own modulus [17]. The latter, in turn, is determined by an integration constant (the Yang-Mills mass scale $\Lambda$ ) and temperature $T$. Apart from small correlations, caused by boundary overlaps/ packing voids of centers as well as peripheries-enabled low-frequency waves and effectively mediated through well-controlled radiative corrections [17, 22], the quantum physics within a given (anti)caloron center is independent of the quantum physics within any other (anti)caloron center. This is the reason why the associated fluctuations, whose spatial range does not exceed $|\phi|^{-1}$, are subject to a Bose-Einstein distribution function. In contrast to the massive sector, whose excitations thus can only fluctuate within each (anti)caloron center separately, the massless sector propagates in a wave-like way for frequencies deeply within the Rayleigh-Jeans regime (the separation between photon-like, localized fluctuations and wave-like long-range propagation is set by a frequency scaling like $T^{-2}$ [20]). Therefore, information on temperature perturbations, carried by the massless sector, are effectively propagated at the speed of light (the average behaviour of localized photonic fluctuations riding the low-frequency waves of the deeply Rayleigh-Jeans regime). Massive fluctuations, on the other hand, do not contribute to the propagation of temperature fluctuations.

The present contribution sketches essential steps in the derivation of a useful a priori estimate of the deconfining thermal ground state, its emergent structure - determinining its excitability in terms of waves and thermal quasiparticles -, and a number of applications to the Cosmic Microwave Background (CMB). In Sec. 2 we review properties of topological-charge-modulus-unity (anti)calorons of trivial (HS) and nontrivial (LLvBK) holonomy along with a discussion of their stability. How the time dependence of the field strength of HS (anti)caloron centers can be trivialised (to become a mere, time dependent choice of gauge) by a spatial coarse-graining over its adjointly transforming two-point correlator is discussed in Sec. 3. In Sec. 4 we review the effective action after coarse-graining, characterized by an inert and adjoint field $\phi$, which breaks $\mathrm{SU}(2)$ to $\mathrm{U}(1)$, and also coarse-grained gauge fields, collectively describing (anti)caloron peripheries, their wave-like excitation, and localized thermal quasiparticle fluctuations which associate with (anti)caloron centers. This theory admits a loop expansion in a completely fixed and physical gauge with no need to introduce ghost fields to reduce the redundancy of perturbative field configurations under linear gauge conditions. Sec. 5 addresses the radiative corrections to the pressure and the polarisation tensor of the massless mode. Concerning the former, the counting of excluded scattering channels and energy-sign combinations of loop momenta vs. a priori possible ones in low-loop-order 2-particle-irreducible bubble diagrams for the pressure, arising solely from massive quasiparticle fluctuations, is performed. Next, an analytical computation of the high- $T$ behaviour of the three-loop diagram is sketched and confronted with numerical results for low $T$. At high $T$ this diagram grows with a power $T^{13}$, and therefore, by itself, does not represent a small correction to the Stefan-Boltzmann limit exhibited by free quasiparticle excitations (one-loop result). This motivates the resummation of all bubble diagrams with dihedral symmetry, the three-loop diagram being the lowest loop order. Together with numerical results for the two-loop diagram this resummation is carried out in terms of the associated Dyson-Schwinger equation, and a well controlled extrapolation to high- $T$ of the low- $T$ behaviour, the latter indicated by the three-loop diagram, is thus 
obtained. We also review earlier results on the one-loop polarisation tensor of the massless mode and its resummation to yield dispersion laws for longitudinally and transversely propagating disturbances. In Sec. 7 we discuss implications for the Cosmic Microwave Background (CMB) once the assumption is made that this thermal photon gas is subject to deconfining $\mathrm{SU}(2)$ rather than $\mathrm{U}(1)$ gauge dynamics. In particular, we emphasize how the Yang-Mills scale of this theory is fixed by radio-frequency CMB observations, the occurrence of a modified temperature-redshift relation in an FLRW Universe, its implications for the cosmological model at high redshifts with a potential resolution of the present discrepancy in $H_{0}$ when extracted from local cosmology and the CMB, radiatively induced anomalies for two-point correlation function at large angles, and the late-time emergence of intergalactic magnetic fields. Finally, in Sec. 8, we summarise our present discussion and provide an outlook on future work. Most definitely, this concerns the resummation of dihedral diagrams involving massless and massive modes, the match between the successful low-redshift $\Lambda$ CDM cosmological model and the new model at high redshifts in terms of percolated and depercolated vortices of a Planck-scale axion, and the according check of viability against the observed angular power spectra of CMB temperature and polarisation correlation functions with a possible dynamical clarification of large-angle anomalies.

\section{2 (Anti)calorons}

The fundamental (Euclidean) SU(2) Yang-Mills action at finite temperature $T$ is

$$
S=\frac{1}{2 g^{2}} \operatorname{tr} \int_{0}^{\beta} d x_{4} d^{3} x F_{\mu \nu} F_{\mu \nu},
$$

where the field-strength tensor $F_{\mu \nu}$ is defined as $F_{\mu \nu}=\partial_{\mu} A_{\nu}-\partial_{v} A_{\mu}+i\left[A_{\mu}, A_{\nu}\right]$ and the gauge field $A_{\mu}$ as $A_{\mu}=A_{\mu}^{a} t_{a}\left(\mu, v=1,2,3,4, a=1,2,3\right.$, and $A_{\mu}^{a}$ real $)$, tr demands tracing over products of matrices in $\operatorname{su}(2), t^{a}$ are hermitian generators (a basis in su(2)) in the fundamental representation $(2 \times 2$ matrices), which are normalised as $\operatorname{tr} t_{a} t_{b}=\frac{1}{2} \delta_{a b}$, and $g$ is the fundamental gauge coupling. (Anti)selfdual configurations $\left(F_{\mu \nu}=\frac{1}{2} \epsilon_{\mu \nu \kappa \lambda} F_{\kappa \lambda}\right.$ with $\epsilon_{1234}=1$ and totally antisymmetric) solve the second-order Yang-Mills equations $D_{\mu} F_{\mu \nu}=0$ (covariant, adjoint derivative defined as $D_{\mu} \cdot=\partial_{\mu} \cdot+i\left[A_{\mu}, \cdot\right]$ ) and, in a given topological sector (homotopy group $\Pi_{3}(\mathrm{SU}(2))=\mathbf{Z}$ ) of topological charge $k \in \mathbf{Z}$ saturate the Bogomoln'yi bound for the Yang-Mills action of Eq. (1):

$$
S=\frac{8 \pi^{2}}{g^{2}}|k| .
$$

As one can easily show [17, 23], (anti)selfdual gauge-field configurations cause the energymomentum tensor $\theta_{\mu \nu}$ of the theory to vanish identically which makes them candidates for the composition of the thermal ground state.

The venue for the construction of periodic configurations with $|k|=1$ was opened by 't Hooft [24] who showed that in the so-called singular gauge, where the $|k|=1$ instanton configuration $A_{\mu}$ on $\mathbf{R}^{4}$ is singular at the peak of its action density [24, 25], the gauge field $A_{\mu}$ for $|k|>1$ can be represented as

$$
A_{\mu}= \begin{cases}\bar{\eta}_{\mu \nu}^{a} t_{a} \partial_{v} \log \Pi(x) & (k>0) \\ \eta_{\mu \nu}^{a} t_{a} \partial_{\nu} \log \Pi(x) & (k<0) .\end{cases}
$$

Here, the scalar function $\Pi$ (pre-potential for $k>1$ ) is a superposition of gauge-field pre-potentials for $k=1$. In Eq. (3) $\bar{\eta}_{\mu \nu}^{a}$ and $\eta_{\mu \nu}^{a}$ denote the antisymmetric-in- $\mu \nu$ and (anti)selfdual 't Hooft symbols (required when decomposing a pure-gauge configuration, winding with $|k|=1$ at spacetime infinity, 
into its su(2) components). This superposition principle for the pre-potential on $\mathbf{R}^{4}$ was exploited by Harrington and Shepard [19] to construct an infinite "mirror sum", based on a "seed" pre-potential centered at $\mathbf{x}=x_{4}=0$ and of instanton scale parameter $\rho$ (a measure for the spread of action density), to achieve periodicity in $x_{4}$ and topological charge $|k|=1$ on $S_{1} \times \mathbf{R}^{3}$. They obtain

$$
\Pi\left(x_{4}, r ; \rho, \beta\right)=1+\frac{\pi \rho^{2}}{\beta r} \frac{\sinh \left(\frac{2 \pi r}{\beta}\right)}{\cosh \left(\frac{2 \pi r}{\beta}\right)-\cos \left(\frac{2 \pi x_{4}}{\beta}\right)},
$$

where $r \equiv|\mathbf{x}|$. It is easy to check that for $r \rightarrow \infty$ the exponential of the line integral of $A_{4}$ along $x_{4}$ (the Polyakov loop) is unity: this configuration is of trivial holonomy. We also note that the function $\Pi$ in Eq. (4) approximates as follows for $|x| \equiv \sqrt{x_{\mu} x_{\mu}} \ll \beta$ and for $r \gg \beta$ [26]:

$$
\Pi=\left\{\begin{array}{cc}
\left(1+\frac{1}{3} \frac{s}{\beta}\right)+\frac{\rho^{2}}{x^{2}} & (|x| \ll \beta) \\
1+\frac{s}{r} & (r \gg \beta),
\end{array}\right.
$$

where the length scale $s$ is given as $s=\frac{\pi \rho^{2}}{\beta}$. For $|x| \ll \beta$ the field strength $F_{\mu \nu}$ is that of an (anti)instanton with a re-scaled parameter $\rho^{\prime 2}=\frac{\rho^{2}}{1+\frac{s}{\beta}}$, for $\beta \ll r \ll s$ one has

$$
B_{i}^{a} \equiv \frac{1}{2} \epsilon_{i j k} F_{j k}^{a}= \pm E_{i}^{a} \equiv \pm F_{4 i}^{a}=\frac{\hat{x}^{a} \hat{x}_{i}}{r^{2}}
$$

and for $\beta \ll s \ll r$

$$
B_{i}^{a}= \pm E_{i}^{a}=s \frac{\delta_{i}^{a}-3 \hat{x}_{i} \hat{x}^{a}}{r^{3}},
$$

where $\hat{x}_{i} \equiv \frac{x_{i}}{r}$ and $\hat{x}^{a} \equiv \frac{x^{a}}{r}$. Since, as we will argue in Sec. 3, the value of the scale parameter $\rho$ is sharply centered at $|\phi|^{-1}$ for all those HS (anti)calorons that contribute to the a priori estimate of the thermal ground state of the deconfining phase and with a lower bound on possible temperatures, judged by the Yang-Mills scale $\Lambda$, one easily shows [20] that the following hierarchy is always satisfied:

$$
\beta \ll|\phi|^{-1} \ll s,
$$

which makes the above considered spatial distance regimes, implying Eqs. (6) ((anti)selfdual, static monopole) and (7) ((anti)selfdual, static dipole), relevant.

At $|k|=1$ (anti)calorons with nontrivial holonomy (Polyakov at spatial infinity not equal to an element of the SU(2) center group $\{-\mathbf{1}, \mathbf{1}\}$ ) are much harder to construct $[27,28]$ via Nahm's beautiful and deep transformation between (anti)selfdual gauge-field configurations on a torus and its dual [29]. Nontrivial holonomy can be prescribed, e.g., by letting $A_{4}\left(r \rightarrow \infty, x_{4}\right)=u t_{3}$ with $0<u<\frac{2 \pi}{\beta}$. Thus $A_{4}$ must be considered an adjoint Higgs field for the spatial components $A_{i}$, inducing, at overall charge neutrality, a pair of a static magnetic monopole and its antimonopole with an exact cancellation between the $A_{4}$-field mediated repulsion and the $A_{i}$-field mediated attraction. This situation can smoothly be connected to trivial holonomy $u \rightarrow 0$ or $u \rightarrow \frac{2 \pi}{\beta}$. Namely, given $\rho$ and $\beta$ the monopoleantimonopole pair is spatially separated by the length scale $s$, and the holonomy $u$ assigns masses to them as $m_{m}=4 \pi u$ and $m_{a}=4 \pi\left(\frac{2 \pi}{\beta}-u\right)(g=1)$. Therefore, trivial holonomy renders one of these defects massless, spreading it over space, while its partner remains massive and localised. (Notice that masslessness for both monopole and antimonopole can also be enforced at nontrivial holonomy if the gauge coupling is sent to infinity $(g \rightarrow \infty)$ in which case both defects become point-like. This can be seen by absorbing $g^{-1}$ into the gauge fields of Eq. (1), see also [30,31].) 
As we will argue in Sec. 3, only (anti)calorons with $|k|=1$ may enter the a priori estimate of the thermal ground state. Accepting this for the moment, the question remains how the holonomy is to be picked. By a heroic deed, Diakonov and collaborators showed in [32] by computing the one-loop quantum weights along the lines of 't Hooft's calculation of the instanton weight [24] that nontrivial holonomy makes the configuration $A_{\mu}$ unstable. Namely, for $\frac{s}{\beta}=\pi\left(\frac{\rho}{\beta}\right)^{2} \gg 1$, which is a relevant limit, see (8), small holonomy $\left(0 \leq u \leq \frac{\pi}{\beta}\left(1-\frac{1}{\sqrt{3}}\right)\right.$ and for $\left.\frac{\pi}{\beta}\left(1+\frac{1}{\sqrt{3}}\right) \leq u \leq 2 \frac{\pi}{\beta}\right)$ perturbes the balance between attraction and repulsion in favour of attraction, while the opposite is true of the situation with large holonomy (complementary range), causing the (anti)caloron to dissociate. As it is easily argued [17], the former situation is much more likely than the latter one. To construct an a priori estimate, however, neither large nor small holonomy are admissible due to the instability of the associated (anti)caloron. This only leaves the HS (anti)caloron as a valid component of the thermal ground-state estimate.

\section{Spatial coarse-graining and a two-point correlator}

Let us now quote the essential steps in deriving the field $\phi$ from a spatial coarse-graining over the following two-point function:

$$
\left\{\hat{\phi}^{a}\right\} \equiv \sum_{C, A} \operatorname{tr} \int d^{3} x \int d \rho t^{a} F_{\mu \nu}(\tau, \mathbf{0})\{(\tau, \mathbf{0}),(\tau, \mathbf{x})\} F_{\mu \nu}(\tau, \mathbf{x})\{(\tau, \mathbf{x}),(\tau, \mathbf{0})\},
$$

where

$$
\{(\tau, \mathbf{0}),(\tau, \mathbf{x})\} \equiv \mathcal{P} \exp \left[i \int_{(\tau, \mathbf{0})}^{(\tau, \mathbf{x})} d z_{\mu} A_{\mu}(z)\right],\{(\tau, \mathbf{x}),(\tau, \mathbf{0})\} \equiv\{(\tau, \mathbf{0}),(\tau, \mathbf{x})\}^{\dagger},
$$

and, from now on, $\tau \equiv x_{4}$. The Wilson lines in Eq. (10) are calculated along the straight spatial line connecting the points $(\tau, \mathbf{0})$ and $(\tau, \mathbf{x})$, and $\mathcal{P}$ demands path-ordering. In (9) the sum is over the $|k|=1 \mathrm{HS}$ caloron $(C)$ and anticaloron $(A)$, and $\left\{\hat{\phi}^{a}\right\}$ signal a family of (dimensionless) phases of the field $\phi$ whose continuous parameters emerge partially in the course of evaluating the right-hand side and partially relate to temporal shift moduli. It is straight-forward to argue [17] that (9) is unique: adjointly transforming one-point functions vanish identically due to (anti)selfduality, higher $n$-point functions and higher topological charges are excluded by dimensional counting, the coincidence of the spatial (anti)caloron center with $\mathbf{0}$ is demanded by spatial isotropy, and the straight-line evaluation of Wilson lines by the absence of any spatial scale on the classical (Euclidean) level. Actually, this allows to ignore the path-ordering prescription since $A_{i}$ is a spatial hedge-hog, centered at $\mathbf{0}$, which assigns to each direction in $\mathbf{R}^{3}$ the same direction in $\operatorname{su}(2)$.

As a result of performing the integrations in (9) it turns out that $\{(\tau, \overrightarrow{0}),(\tau, \vec{x})\}$ is characterized by harmonic motion of period $\beta$ within some plane $\mathbf{R}^{2} \subset \operatorname{su}(2)$ (global gauge choice) subject to unspecified normalizations and phases for the oscillations along each of the two axes [33]. Therefore, $\left\{\hat{\phi}^{a}\right\}$ uniquely comprises the kernel of the linear second-order operator $\mathcal{D} \equiv \partial_{\tau}^{2}+\left(\frac{2 \pi}{\beta}\right)^{2}$. Because (anti)caloron action is independent of temperature, however, the explicit temperature dependence in $\mathcal{D}$ must be absorbed into the $\phi$-derivative of a potential $V$. Demanding $\phi$ to be simultaneously the solution of a first-order BPS and the Euler-Lagrange equation, one derives the following first-order equation for $V$ [17]

with solution

$$
\frac{\partial V\left(|\phi|^{2}\right)}{\partial|\phi|^{2}}=-\frac{V\left(|\phi|^{2}\right)}{|\phi|^{2}}
$$

$$
V\left(|\phi|^{2}\right)=\frac{\Lambda^{6}}{|\phi|^{2}}
$$


where $\Lambda$ denotes an arbitrary mass scale (the Yang-Mills scale). Since the BPS equation, which exhibits the square root of $V$ and demands "circular polarisation" for the harmonic motion in the plane, needs to be satisfied in addition to the Euler-Lagrange equation (first derivative of $V$ ) the usual additive shift symmetry of the potential in the Euler-Lagrange equation no longer is an option: Should $V=4 \pi \Lambda T^{3}$ (with $|\phi|=\sqrt{\frac{\Lambda^{3}}{2 \pi T}}$ ) alone turn out to be a good a priori estimate of the thermal ground state's energy density, see Sec. 4 , then this result is unique.

It is important to note that the integration over the instanton scale parameter $\rho$ depends cubically on an upper integration limit $\rho_{u}$ and that the dependence on $\tau$ of this integral saturates very rapidly for $\rho_{u} / \beta>1$ into the harmonic one. Therefore, the kernel $\left\{\hat{\phi}^{a}\right\}$ in Eq. (9) of the differential operator $\mathcal{D}$ is strongly dominated by a small band of $\rho$ values centered at the cutoff $\rho_{u}=|\phi|^{-1}$, and one can show that $\rho_{u} / \beta=|\phi|^{-1} / \beta \gg 1$ for all temperatures within the deconfining phase [17].

\section{Effective action, adjoint gauge-symmetry breaking, waves, and thermal quasiparticles}

Since $\phi$ is inert (no momentum transfer to and from this field) and the Yang-Mills action, restricted to topologically trivial gauge fields, is renormalisable [34] and since the effective Lagrangian density $\mathcal{L}_{\text {eff }}$ (after spatial coarse-graining) is required to be gauge invariant, one arrives at the following, unique answer [17]

$$
\mathcal{L}_{\text {eff }}\left[a_{\mu}\right]=\operatorname{tr}\left(\frac{1}{2} G_{\mu \nu} G_{\mu \nu}+\left(D_{\mu} \phi\right)^{2}+\frac{\Lambda^{6}}{\phi^{2}}\right),
$$

where $G_{\mu \nu}=\partial_{\mu} a_{\nu}-\partial_{\nu} a_{\mu}-i e\left[a_{\mu}, a_{\nu}\right] \equiv G_{\mu \nu}^{a} t_{a}$ denotes the field strength of the effective trivial-topology gauge field $a_{\mu}=a_{\mu}^{a} t_{a}, D_{\mu} \phi=\partial_{\mu} \phi-i e\left[a_{\mu}, \phi\right]$, and $e$ is the effective gauge coupling. A solution $a_{\mu}^{\mathrm{gs}}$ to the effective, second order Yang-Mills equations with $D_{\mu} \phi=G_{\mu \nu}=0$ reads $a_{\mu}^{\mathrm{gs}}=\delta_{\mu 4} \frac{2 \pi}{e \beta} t_{3}$ if without restriction of generality (global gauge choice) circular polarisation in the 1-2 plane of $\operatorname{su}(2)$ is considered for $\phi$. On $\phi$ and $a_{\mu}^{\mathrm{gs}}$ the action density (13) thus reduces to the potential $\operatorname{tr} V$, and the vanishing energy density and pressure of (anti)caloron centers is made finite by (anti)caloron overlap, effectively represented by $a_{\mu}^{\mathrm{gs}}$ :

$$
\rho^{\mathrm{gs}}=-P^{\mathrm{gs}}=4 \pi \Lambda^{3} T .
$$

On $a_{\mu}^{\mathrm{gs}}$ the Polyakov loop turns out to be $\mathbf{- 1}$, and one can show that a singular but admissible (time periodic) gauge rotation exists which transforms $\phi$ to unitary gauge $\phi=2|\phi| t_{3}$ and $a_{\mu}^{\mathrm{gs}}$ to $a_{\mu}^{\mathrm{gs}}=0$ where the Polyakov loop now is $\mathbf{1}$. This demonstrates the electric $\mathbf{Z}_{2}$ degeneracy of the thermal ground-state estimate - a sure sign of deconfinement.

In unitary gauge one can read off from the action density (13) the mass spectrum $m_{a}^{2}=$ $-2 e^{2} \operatorname{tr}\left[\phi, t_{a}\right]\left[\phi, t_{a}\right]$ for the gauge-field excitations as induced by the adjoint Higgs mechanism:

$$
\begin{aligned}
& m^{2} \equiv m_{1}^{2}=m_{2}^{2}=4 e^{2} \frac{\Lambda^{3}}{2 \pi T}, \\
& m_{3}=0 .
\end{aligned}
$$

If, instead, one appeals to the Dyson series for mass generation then one can easily show that any attempt to move a massive gauge-field fluctuation away from its mass shell would inevitably transfer energy-momentum to the field $\phi$. This, however, would contradict the very derivation of $\phi$. Thus massive quasiparticle fluctuations, albeit deeply probing and thus orginated by (anti)caloron centers (quantum excitation, no classical or quasi-classical wave propagation: $e \geq \sqrt{8} \pi \Rightarrow m=\omega \gg|\phi|$, see below, where $\omega$ is the circular would-be frequency of a wave-like propagation, compare with [20] for 
the massless case $m_{3}=0$ ) need to be understood as Bose-Einstein distributed quasiparticle on-shell (quantum) excitations.

By imposing thermodynamical selfconsistency onto the thermal ground-state estimate and its free thermal quasiparticle excitations (intactness of Legendre transformation between thermodynamical quantities as computed in the theory given by Eq. (13)) one derives the following evolution equation for the effective gauge coupling $e$

$$
1=-\frac{24 \lambda^{3}}{(2 \pi)^{6}}\left(\lambda \frac{d a}{d \lambda}+a\right) a D(2 a)
$$

where $D(y) \equiv \int_{0}^{\infty} d x \frac{x^{2}}{\sqrt{x^{2}+y^{2}}} \frac{1}{\mathrm{e}^{\sqrt{x^{2}+y^{2}}-1}}, a \equiv \frac{m}{2 T}$, and $\lambda \equiv \frac{2 \pi T}{\Lambda}$. Lowering $\lambda$ from a sufficiently high initial value $\lambda_{i}$ the solutions to Eq. (16), when solved for $e(\lambda)$, linearly fast run into the attractor $e=\sqrt{8} \pi$ for $\lambda_{i} \gg \lambda \gg \lambda_{c}=13.87$ and $e \propto-\log \left(\lambda-\lambda_{c}\right)$ for $\lambda \sim \lambda_{c}$ [17]. Since only (anti)calorons with $\rho \sim|\phi|^{-1}$ contribute to the emergence of field $\phi$ one can use $g=e$ in the (anti)caloron action (2) $(|k|=1)$. Moreover, by re-instating $\hbar$ in the effective action, one easily arrives at $e=\frac{\sqrt{8} \pi}{\sqrt{\hbar}}[17,21,35]$, and thus $S=\hbar$. At $\lambda_{c}$ the magnetic (anti)monopoles liberated by rarely occurring dissociations of largeholonomy (anti)calorons - an effectively described radiative effect $[17,36,37]$ - become point-like and massless.

For the estimate of thermodynamical quantities based on free thermal quasiparticle fluctuations to be reliable, it must be assured that radiative (nonthermal) corrections remain small. The next Section provides results indicating that, modulo possible resummations, this indeed is the case.

\section{Radiative corrections: Massive sector}

Here we only consider higher loop corrections involving massive thermal quasiparticle fluctuations. The discussion is a summary of the results obtained in [22] and shows that, in general, radiative corrections to the pressure in the deconfining phase, although small and well under control for all temperatures, are not subject to a thermodynamical interpretation.

\subsection{Counting excluded vs. allowed scattering-channel and energy-sign combinations in bubble diagrams}

In [22] a systematic study of 2-particle-irreducible (2PI) bubble diagrams with massive lines only, which radiatively contribute to the loop expansion of the pressure in the deconfining phase, was performed. The region of integration over loop momenta is severely constrained by the demand that in each scattering channel for a given four-vertex the invariant momentum transfer (Mandelstam variables $s, T$, and $u$ ) is bounded by $|\phi|^{2}$ in unitary-Coulomb gauge. A measure of how constrained loop momenta turn out to be is obtained by comparing the a priori (without constraints) possible combinations of their energy signs in a given diagram with the allowed combinations after imposing the constraints. The ratio $R(L)$ of the latter to the former in dependence of loop order $L$ maximally is

$$
R(3)=0.1667, R(4)=0.0463, R(5)=0.0123, R(6)=0.0044 .
$$

Multiplying $R$ with the symmetry factor $S$ of a diagram maximally yields

$$
R \cdot S(3)=0.00347, R \cdot S(4)=0.000965, R \cdot S(5)=0.000386, R \cdot S(6)=0.000113 \text {. }
$$


Eqs. (17) and (18) testify of an impressive suppression of allowed loop fluctuations with increasing loop order as imposed by the vertex constraints. Yet, one can demonstrate that diagrams with dihedral symmetry (polygons inscribed into the circle with corners defining four-vertices) will exhibit allowed energy-sign combinations at an arbitrarily high loop order. This motivates the explicit computation of the dihedral diagram with $L=3$.

\subsection{Low- and high-temperature behavior of three-loop diagram}

The three-loop diagram in Fig. 1 is subject to the following expression [22]

$$
\begin{aligned}
\left.\Delta P\right|_{3-\text { loop }}=i \frac{\Lambda^{4}}{48 \lambda^{2}} e^{4} \frac{1}{(2 \pi)^{6}} \sum_{\text {signs }} \int d \theta_{1} d \varphi_{1} d r_{1} d r_{2} d \theta_{3} \sum_{\left\{r_{3}\right\}} r_{1}^{2} r_{2}^{2} r_{3}^{2} \sin \theta_{1} \sin \theta_{3} & \\
& \times P\left(p_{i}\right) \frac{n_{B}^{\prime}\left(r_{1}\right) n_{B}^{\prime}\left(r_{2}\right) n_{B}^{\prime}\left(r_{3}\right) n_{B}^{\prime}\left(r_{4}\right)}{8\left|p_{1}^{0} p_{2}^{0} p_{3}^{0} p_{4}^{0}\right|} .
\end{aligned}
$$

The first sum in (19) runs over allowed sign combinations for $p_{i}^{0}, i=1, \ldots, 4$, we have scaled all momentum components $p_{i}^{\mu}$ and mass $m$ dimensionless by $|\phi|, n_{B}^{\prime}\left(r_{i}\right) \equiv n_{B}\left(2 \pi \sqrt{\frac{r_{i}^{2}+m^{2}}{\lambda^{3}}}\right)\left(n_{B}\right.$ the Bose-Einstein distribution), $P\left(p_{i}\right)$ denotes a certain polynomial of invariants formed by the loop fourmomenta $p_{i}, r_{i}$ denotes the modulus of the spatial momentum $\mathbf{p}_{i}$, and angular variables parametrise their mutual orientation. The second sum in (19) runs over all solutions in $r_{3}$ of the equation

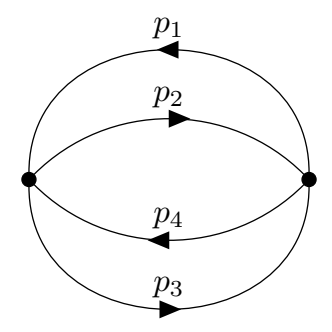

Figure 1. Dihedral diagram with $L=3$.

$$
\begin{aligned}
\operatorname{sgn}\left(p_{2}^{0}\right) \sqrt{r_{2}^{2}+m^{2}}+\operatorname{sgn}\left(p_{3}^{0}\right) \sqrt{r_{3}^{2}+m^{2}}-\sqrt{r_{1}^{2}+m^{2}}=-\left[r_{1}^{2}+r_{2}^{2}+r_{3}^{2}\right. \\
\left.\quad-2 r_{1} r_{2} \cos \theta_{1}-2 r_{1} r_{3}\left(\sin \varphi_{1} \sin \theta_{1} \sin \theta_{3}+\cos \theta_{1} \cos \theta_{3}\right)+2 r_{2} r_{3} \cos \theta_{3}+m^{2}\right]^{1 / 2} .
\end{aligned}
$$

While an analytical treatment of (19) is impossible at low temperatures and therefore requires MonteCarlo integration one can, however, extract the leading power in $\lambda$ in a high-temperature expansion. Namely, one obtains

$$
\begin{aligned}
\left.\Delta P\right|_{3-\text { loop }} & =i \frac{\Lambda^{4}}{\lambda^{2}} e^{4} \frac{1}{(2 \pi)^{5}} \frac{1}{15}\left(1+\frac{1}{4 m^{2}}\right) \frac{I_{3}^{2}}{m^{8}} \\
& =i \Lambda^{4} \frac{1}{3375} \frac{1}{(2 \pi)^{15}} \frac{1}{m^{4}}\left(1+\frac{1}{4 m^{2}}\right)\left(\pi^{4}-90 \zeta(5)\right)^{2} \lambda^{13} \\
& =5.2968 \cdot 10^{-20} i \Lambda^{4} \lambda^{13}
\end{aligned}
$$


Eq. (21) is the result of an elaborate analysis which is confirmed by the numerical computation. The latter predicts an impressive downward hierarchy between one-loop, two-loop, and three-loop contributions at small temperatures. The power of thirteen at high temperatures is strongly indicative that a resummation of all dihedral loop orders is required since the Stefan-Boltzmann power of the one-loop contribution to the pressure exhibits a power of four only. Notice that $\left.\Delta P\right|_{3 \text {-loop }}$ is dominated by a purely imaginary contribution at high temperatures.

\subsection{Resummation of dihedral diagrams}

The resummation of dihedral diagrams to all loop orders is performed in terms of the solution of the according Dyson-Schwinger equation for the vertex form factor $f(\lambda)$ at high temperatures. One obtains [22]

$$
\begin{aligned}
\left.f^{2}(\lambda) \Delta P\right|_{3-\text { loop }} & \approx\left(-0.94 \cdot 10^{15} i \lambda^{-11.6}\right)^{2} \cdot 5.3 \cdot 10^{-20} i \Lambda^{4} \lambda^{13} \\
& =-4.7 \cdot 10^{10} i \Lambda^{4} \lambda^{-10.2} .
\end{aligned}
$$

Thus, upon resummation the power of thirteen of the "naked" three-loop diagram has dropped to a power of roughly minus ten as a result of resummation. One can also show that the leading powers of the resummed two-loop and three-loop diagrams cancel. In general, this does not imply the cancellation of subleading powers, and one should count on the appearance of imaginary contributions. Physically, these introduce small nonthermal behavior (turbulences) which can be envisaged corrections to the isotropic and homogeneous a priori estimate of the thermal ground-state field $\phi$ in terms of a single (anti)caloron center as introduced by slight overlaps and packing voids.

\section{Radiative corrections: Massless sector}

In addition to an analysis of feeble one-loop photon-photon scattering in [38] and the two-loop bubble diagrams involving massless modes [39], which contribute to the pressure, we have computed the polarisation tensor of the massless mode at resummed one-loop order in [36, 40] and in [41]. This requires the solution of gap equations for the screening functions $G$ and $F$ for transverse and longitudinal photons, respectively. Briefly, function $G$ predicts a low-frequency gap (screening) in the spectral intensity (radiance) of a black body which closes like $\lambda^{-1 / 2}$ with increasing temperature and is absent at $\lambda_{c}$. Shortly above the gap a localised spectral region of antiscreening is encountered. Function $F$, on the other hand, associates with three branches of low-momentum longitudinal modes (charge-density waves).

\section{Implications for the Cosmic Microwave Background (CMB) and the Cosmological Model}

There are various implications of deconfining SU(2) Yang-Mills thermodynamics for the CMB once the postulate is made that it describes thermal photon gases with an electric-magnetic dual interpretation of its Cartan subalgebra [17]. The latter property is easily derived from $e=\frac{\sqrt{8} \pi}{\sqrt{\hbar}}$, see Sec. 4 , and the fact that in the same system of units $\left(c=1=\epsilon_{0}=\mu_{0}\right)$ the fine-structure constant of QED reads $\alpha=\frac{Q^{2}}{4 \pi \hbar}$ since this implies that the electric charge $Q$ is inversely proportional to effective Yang-Mills coupling $e$. Since $g=\frac{4 \pi}{e}$ is the charge of a magnetic monopole in SU(2) Yang-Mills thermodynamics this means that electric charges in the real world are magnetic charges w.r.t $\mathrm{U}(1) \subset \mathrm{SU}(2)$ and vice versa. 
In particular, such a description should apply to the CMB. In [42] an excess of spectral CMB power detected from about $3 \mathrm{GHz}$ [43] down to $40 \mathrm{MHz}$ [44] was interpreted as the onset of electric monopole condensation and the associated Meissner effect (evanescence of low-frequency waves). This, however, can only be the case if $T_{c}$ practically coincides with the present CMB baseline temperature $T_{0}=2.725 \mathrm{~K}$, setting the Yang-Mills scale to $\Lambda_{\mathrm{CMB}} \sim 10^{-4} \mathrm{eV}$.

Let us mention a few consequences. First, an SU(2) Yang-Mills theory of this scale implies a modified temperature $(T)$ - redshift $(z)$ relation in an FLRW universe [46]. Namely, there is curvature in this relation at low $z$, and at high $z$ one has $T / T_{0}=0.63(z+1)$. There are two immediate physics implications: (i) resolution of the re-ionisation puzzle (high $z_{\text {re }}$ from extraction using the CMB angular power spectra, low $z_{\text {re }}$ from detection of Gunn-Peterson trough in quasar spectra [45]) [46], (ii) drastic change of cold matter content in high- $z$ cosmological model [47] since recombination now occurs at a considerably higher redshift with a resolution of the $H_{0}$ (today's value of Hubble parameter) puzzle (low $H_{0}$ from extraction using the $\mathrm{CMB}$ angular power spectra, high $H_{0}$ from local cosmological observation (standard candles, comoving sound horizon at baryon drag in matter correlation functions, absolute distance calibrations [48]; time structure of gravitationally lensed quasar light [49]). Second, due to a nontrivial screening function $G$ a cosmologically local depression in the temperature distribution of the CMB is dynamically generated [50-52] which affects the CMB dipole (besides a kinematic contribution arising from the Doppler effect [53]), the low lying multipoles in the $T T$ and other correlation functions [54] (large-angle anomalies), and could invoke the late-time emergence of intergalactic magnetic fields [55] through longitudinal modes, described by screening function $F$ [41], subject to dynamical breaking of statistical isotropy [52].

\section{Summary and Conclusions}

This contribution to ICNFP 2017 has sketched the nonperturbative physics in the deconfining phase of SU(2) Yang-Mills thermodynamics and outlined a few applications towards the CMB, the cosmological model, and the late-time emergence of intergalactic magnetic fields. In particular, the computation of the CMB power spectra within the new cosmological model and taking into account radiative effects in SU(2) Yang-Mills thermodynamics (large-angle anomalies) is under way, and the "microscopic" physics (the relevant spatial distances are typical radii of spiral galaxies) for the percolation/depercolation transition of vortices in a Planck-scale axion field [56, 57] should be understood. Such a transition is required to interpolate the new high- $z$ cosmological model to successful, low- $z$ $\Lambda \mathrm{CDM}$ cosmology [47].

\section{References}

[1] D. J. Gross and F. Wilczek, Phys. Rev. Lett. 30, 1343 (1973).

[2] H. D. Politzer, Phys. Rev. Lett. 30, 1346 (1973).

[3] I. B. Khriplovich, Sov. J. Nucl. Phys. 10, 235 (1970).

[4] I. B. Khriplovich, Yad. Fiz. 10, 409 (1969).

[5] G. 't Hooft, unpublished (1973).

[6] G. 't Hooft, Phys. Rev. Lett. 37, 8 (1976).

[7] T. Banks and A. Casher, Nucl. Phys. B 169, 103 (1980).

[8] G. 't Hooft, Nucl. Phys. B 138, 1 (1978).

[9] S. Mandelstam, Phys. Rep. C 23, 245 (1976).

[10] S. L. Adler and W. A. Bardeen, Phys. Rev. 182, 1517 (1969). 
[11] J. S. Bell and R. Jackiw, Nuovo Cim. A 60, 47 (1977).

[12] K. Fujikawa, Phys. Rev. Lett. 42, 1195 (1979).

K. Fujikawa, Phys. Rev. D 21, 2848 (1980), Erratum-ibid. Phys. Rev. D, 22, 1499 (1980).

[13] P. W. Anderson, Phys. Rev. 130, 439 (1963).

[14] G. S. Guralnik et al., Phys. Rev. Lett. 13, 585 (1964).

[15] F. Englert and R. Brout, Phys. Rev. Lett. 13, 321 (1964).

[16] P. W. Higgs, Phys. Rev. Lett. 13, 508 (1964).

[17] R. Hofmann, The thermodynamics of quantum Yang-Mills theory: Theory and applications (World Scientific, Singapore, 2016).

[18] R. Hofmann, Int. J. Mod.Phys. A 20, 4123 (2005), Erratum-ibid. A 21 (2006) 6515.

[19] B. J. Harrington and H. K. Shepard, Phys. Rev. D 17, 2122 (1978).

[20] T. Grandou and R. Hofmann, Adv. Math. Phys. 2015, Article ID 197197, doi:10.1155/2015/197197 (2015).

[21] D. Kaviani and R. Hofmann, Quantum Matter 2012, 1, 41 (2012).

[22] I. Bischer, T. Grandou, and R. Hofmann, Int. J. Mod. Phys. A 32 no.19n20, 1750118 (2017).

[23] E. Shuryak and T. Schaefer, Rev. Mod. Phys. 70, 323 (1996).

[24] G. 't Hooft, Phys. Rev. D 14, 3432 (1976), Erratum-ibid. D 18, 2199 (1978).

[25] R. Jackiw and C. Rebbi, Phys. Rev. D 14, 517 (1976).

[26] D. J. Gross, R. D. Pisarski, and L. G. Yaffe, Rev. Mod. Phys. 53, 43 (1981).

[27] K. Lee and C. Lu, Phys. Rev. D 58, 025011-1 (1998).

[28] T. C. Kraan and P. Van Baal, Phys. Lett. B 428, 268 (1998).

T. C. Kraan and P. Van Baal, Nucl. Phys. B 533, 627 (1998).

[29] W. Nahm, Phys. Lett. B 90, 413 (1980).

W. Nahm, All self-dual multimonopoles for arbitrary gauge groups, CERN preprint TH-3172, (1981).

W. Nahm, Self-dual monopoles and calorons, in Trieste Group Theor. Method 1983, p. 189, (1983).

[30] G. 't Hooft, Nucl. Phys. B 79, 276 (1974).

[31] M. K. Prasad and C. M. Sommerfield, Phys. Rev. Lett. 35, 760 (1975).

[32] D. Diakonov, N. Gromov, V. Petrov, and S. Slizovskiy, Phys. Rev. D 70, 036003 (2004).

[33] U. Herbst and R. Hofmann, ISRN High Energy Phys. 2012, 373121, doi:10.5402/2012/373121 (2012).

[34] G. 't Hooft, Nucl. Phys. B 33, 173 (1971).

G. 't Hooft and M. Veltman, Nucl. Phys. B 44, 189 (1972).

G. 't Hooft and M. Veltman, Nucl. Phys. B 50, 318 (1972).

[35] S. J. Brodsky and P. Hoyer, Phys. Rev. D 83, 045026 (2011).

J. Iliopoulos, C. Itzykson, and A. Martin, Rev. Mod. Phys. 47, 165 (1975).

J. F. Donoghue et al., Phys. Lett. B 529, 132 (2002).

N. E. J. Bjerrum-Bohr, J. F. Donoghue, and B. R. Holstein, Phys. Rev. D 68, 084005 (2003), Erratum-ibid. Phys. Rev. D 71, 069904 (2005).

B. R. Holstein and J. F. Donoghue, Phys. Rev. Lett. 93, 201602 (2004).

[36] J. Ludescher and R. Hofmann, Annalen d. Physik 18, 271 (2009).

[37] J. Ludescher et al., Annalen d. Physik 19, 102 (2010).

[38] N. Krasowski and R. Hofmann, Annals Phys. 347, 287 (2014).

[39] M. Schwarz, R. Hofmann, and F. Giacosa, Int. J. Mod. Phys. A 22, 1213 (2007).

[40] C. Falquez, R. Hofmann, and T. Baumbach, Annalen d. Physik 522, 904 (2010). 
[41] C. Falquez, R. Hofmann, and T. Baumbach, Quantum Matter 2012, 1, 153 (2012).

[42] R. Hofmann, Annalen Phys. 18, 634 (2009).

[43] D. J. Fixsen et al., Astrophys. J 734, 5 (2011).

[44] P. Reich and W. Reich, Astron. \& Astrophys. Suppl. Ser. 63, 205 (1986).

R. S. Roger et al., Astron. \& Astrophys. Suppl. Ser. 137, 7 (1999).

K. Maeda, et al., Astron. \& Astrophys. Suppl. Ser. 140, 145 (1999).

C. G. T. Haslam, Astron. \& Astrophys. 100, 209 (1981).

[45] R. H. Becker et al., Astron. J 122, 2850 (2001).

[46] R. Hofmann, Annalen d. Phys. 527, 254 (2015).

[47] S. Hahn and R. Hofmann, Mon. Not. Roy. Astron. Soc. 469 no.1, 1233 (2017).

S. Hahn and R. Hofmann, Adv. High Energy Phys. 2017, 7525121 (2017).

[48] J.-L. Bernal, L. Verde, A. G. Riess, JCAP 10, 019 (2016).

A. G. Riess et al., Astrophys. J. 826, 56 (2016).

[49] V. Bonvin et al., Mon. Not. Roy. Astron. Soc. 465 no.4, 4914 (2017).

[50] M. Szopa and R. Hofmann, JCAP 0803, 001 (2008).

[51] J. Ludescher and R. Hofmann, arXiv:0902.3898 (2009).

[52] R. Hofmann, Nature Phys. 9, 686 (2013).

[53] P. J. E. Peebles and T. Wilkinson, Phys. Rev. 174, 2168 (1968).

[54] D. J. Schwarz, C. J. Copi, D. Huterer, and G. D. Starkman, Class. Quant. Grav. 33 no.18, 184001 (2016).

C. J. Copi, D. Huterer, D. J. Schwarz, and G. D. Starkman, Mon. Not. Roy. Astron. Soc. 449 no.4, 3458 (2015).

C. J. Copi, D. Huterer, D. J. Schwarz, and G. D. Starkman, Mon. Not. Roy. Astron. Soc. 451 no.3, 2978 (2015).

C. J. Copi, D. Huterer, D. J. Schwarz, and G. D. Starkman, Mon. Not. Roy. Astron. Soc. 434, 3590 (2013).

[55] P. P. Kronberg, Rept. Prog. Phys., 57, 325 (1994).

A. Neronov and I. Vovk, Science 328, 73 (2010).

L. M. Widrow, Rev. Mod. Phys. 74, 775 (2002).

[56] F. Giacosa and R. Hofmann, Eur. Phys. J. C 50, 635 (2007).

[57] F. Giacosa, R. Hofmann, and M. Neubert, JHEP 0802, 077 (2008). 